\title{
National Neonatal Sepsis Task Force launch: Supporting infection prevention and surveillance, outbreak investigation and antimicrobial stewardship in neonatal units in South Africa
}

A Dramowski, ${ }^{1}$ MB ChB, PhD, FCPaed (SA), MMed (Paediatrics), Cert ID (SA) Paed, DCH (SA); S Velaphi, ${ }^{2}$ MB ChB, MMed (Paediatrics), FCPaed (SA), Fellowship in Perinatal and Neonatal Medicine (UTSW), PhD; G Reubenson, ${ }^{3}$ MB BCh, FCPaed (SA), DCH (SA), DTM\&H; A Bekker, ${ }^{4} \mathrm{MB}$ ChB, PhD, FCPaed (SA), MMed (Paediatrics), Cert Neonatology (SA), DCH (SA); O Perovic, ${ }^{5} \mathrm{MD}$, FCPath (SA) Micro, MMed (Microbiology), DTM\&H; H Finlayson, ${ }^{1}$ MB ChB, FCPaed (SA), Cert ID (SA) Paed, DCH (SA); A Duse, ${ }^{6}$ MT, MB BCh, DTM\&H, MSc Med, MMed (Microbiology), FCPath (SA) Micro; N R Rhoda, ${ }^{7}$ MB ChB, FCPaed (SA), Cert Neonatology (SA); N P Govender, ${ }^{5}$ MB BCh, FCPath (SA) Micro, MMed (Microbiology), MSc (Mycol), DTM\&H, Dip HIV Man (SA), MSc (Epi), FECMM

${ }^{1}$ Division of Paediatric Infectious Diseases, Department of Paediatrics and Child Health, Faculty of Medicine and Health Sciences, Stellenbosch University, Cape Town, South Africa

${ }^{2}$ Department of Paediatrics and Child Health, Faculty of Health Sciences, University of the Witwatersrand, Johannesburg, South Africa

${ }^{3}$ Empilweni Service and Research Unit, Department of Paediatrics and Child Health, Faculty of Health Sciences, University of the Witwatersrand, Johannesburg, South Africa

${ }^{4}$ Division of Neonatology, Department of Paediatrics and Child Health, Faculty of Medicine and Health Sciences, Stellenbosch University, Cape Town, South Africa

${ }^{5}$ National Institute for Communicable Diseases, a Division of the National Health Laboratory Service and School of Pathology, University of the Witwatersrand, Johannesburg, South Africa

${ }^{6}$ Division of Hospital Epidemiology and Infection Control, Clinical Microbiology and Infectious Diseases, School of Pathology, National Health Laboratory Service and University of the Witwatersrand, Johannesburg, South Africa

${ }^{7}$ Department of Neonatology and Paediatrics, Faculty of Health Sciences, University of Cape Town, South Africa

Corresponding author: A Dramowski (dramowski@sun.ac.za)

Despite a substantial decline in childhood mortality rates in South Africa (SA), progress in neonatal mortality reduction has been much slower. Severe bacterial infections remain a leading cause of neonatal morbidity and a direct cause of $13.1 \%$ of neonatal deaths among babies $>1 \mathrm{~kg}$. The incidence of hospital-acquired infections, antimicrobial resistance and outbreaks of infections in SA neonatal units is substantial, and is possibly higher than the currently available estimates. The SA Neonatal Sepsis Task Force was launched in Port Elizabeth, SA, on 13 September 2019 to provide technical advice and guidance on surveillance for neonatal sepsis, infection prevention, case management, antimicrobial stewardship and containment of neonatal unit outbreaks.

S Afr Med J 2020;110(5):360-363. https://doi.org/10.7196/SAMJ.2020.v110i5.14564

\section{Burden of neonatal infection}

Despite a substantial decline in childhood mortality rates in South Africa (SA) ${ }^{[1]}$ and globally, ${ }^{[2]}$ progress in neonatal mortality reduction has been much slower. Severe bacterial infections remain a leading cause of neonatal morbidity and mortality in sub-Saharan Africa, affecting nearly 1 million neonates and causing 250000 deaths annually. ${ }^{[2]}$ At the current rate of decline the United Nations Sustainable Development Goal of reducing neonatal mortality rates to $<12$ deaths per 1000 live births by 2030, will not be attained by many African countries. ${ }^{[3]}$ In SA, infections were estimated to cause $13.1 \%$ of neonatal deaths among babies $>1 \mathrm{~kg}$ from 2012 to 2016 (Perinatal Problem Identification Programme (PPIP) data). ${ }^{[1]}$ However, recent postmortem minimally invasive tissue sampling (MITS) data from the largest neonatal unit in SA (Chris Hani Baragwanath Academic Hospital, Johannesburg) demonstrate that hospitalacquired, antimicrobial-resistant infections are now the leading cause of late neonatal mortality (after the first week of life). These hospital-acquired infections (HAIs) and infection-attributable deaths disproportionately affect preterm and low-birthweight neonates, and are responsible for $58 \%$ of deaths overall v. $70 \%$ of deaths in preterm infants. ${ }^{[4]}$ Attributing cause of death with certainty, particularly in preterm infants, is difficult, and may lead to substantial underestimation of the true burden and impact of neonatal infection in SA.

\section{Challenges in diagnosis and surveillance of neonatal infection}

Major challenges in diagnosing severe neonatal bacterial infections persist. These include the nonspecific clinical presentation of sepsis, inequitable access to microbiology laboratory services, high blood culture contamination rates and low pathogen yields from submitted specimens. Importantly, blood culture, the current gold standard for confirming bacteraemia, has a sensitivity of $\sim 30 \%$, much lower than molecular testing or postmortem MITS. The lack of validated clinical 
definitions of neonatal sepsis further hampers efforts to quantify the neonatal infection burden. The World Health Organization (WHO) recommends using a combination of clinical signs to diagnose possible serious bacterial infection ( $\mathrm{pSBI}$ ), with a reported sensitivity of $85 \%$ and specificity of $75 \%{ }^{[5]}$ However, even in the presence of these clinical signs, infection can still only be confirmed by blood culture. In Africa, there have been limited efforts to pool and harmonise existing laboratory data on neonatal infection, with not a single Anglophone country having population-level estimates of infection burden. ${ }^{[6]}$ Despite the availability of better healthcare and information technology infrastructure, until recently no national surveillance programme existed for invasive neonatal infections, antimicrobial resistance among isolates and neonatal unit outbreaks of infection in SA.

\section{Measurement of infection-attributable} mortality and morbidity

More accurate attribution of neonatal deaths to infection is key. Many infection-related preterm deaths are possibly 'mislabelled' as prematurity related, owing to a lack of accurate diagnostics. ${ }^{[4,7]}$ Death notification forms, the basis of burden of disease and mortality studies, often indicate only the underlying cause rather than the immediate cause of death, resulting in underestimation of infectionrelated deaths in preterm infants. Current SA neonatal mortality data $^{[1]}$ do not account for deaths in extremely-low-birthweight babies $(<1000 \mathrm{~g})$ and babies who remain hospitalised in a neonatal unit beyond 28 days of life. Both these groups are at high risk of infection, and therefore should be included in neonatal unit surveillance activities as sentinel populations to serve as indicators of the impact of infection. Another important omission in current infection surveillance, is the failure to measure and report prolonged hospitalisation, repeated and prolonged antibiotic use, hospital costs, infection complications and lifelong neurodevelopmental disability related to neonatal infections.

\section{Threat of hospitall-acquired infection and antimicrobial resistance}

PPIP cites HAIs as the second most prevalent avoidable factor in neonatal deaths. ${ }^{[1]}$ Bloodstream infections are the most frequent HAI type in neonates, with infection rates varying from 4 to 14 cases per 1000 patient-days, although data are available from only a few large institutions. ${ }^{[8-13]}$ Similarly, neonatal infection outbreaks in SA are common, but are rarely reported. They are likely to be more frequent in units with overcrowding, understaffing, sharing of equipment and poor environmental cleaning. ${ }^{[14,15]}$ The rise in antimicrobial resistance (AMR) among hospital pathogens globally is of concern for neonatal units, particularly those in Africa, where Gram-negative bacterial pathogens predominate. ${ }^{[8-12]}$

Increases in AMR rates threaten the continued effectiveness of currently recommended first-line antibiotics, i.e. ampicillin/penicillin and gentamicin. Further, the epidemiology of neonatal sepsis seems to be changing, with AMR infections often occurring in the first days of life, possibly owing to vertical transmission following maternal AMR colonisation during antenatal care or labour and delivery. This necessitates early use of broad-spectrum, secondline antibiotics, which further increases the risk of neonatal AMR bacterial colonisation and recurrent infections. Treatment options are limited for certain pathogens, e.g. carbapenem-resistant Acinetobacter baumannii (CRAB) and Enterobacteriaceae (CRE), which are the leading HAI pathogens in several SA neonatal units..$^{[4,12]}$ The true burden and impact of AMR in SA neonatal units remain largely unknown, because of a lack of national laboratory-based neonatal infection surveillance, limited detection and reporting of outbreaks and an absence of HAI surveillance programmes.

\section{Factors contributing to hospital- acquired infections and antimicrobial resistance}

Hospitalised neonates are a vulnerable population owing to their immature immune systems, acquisition of multidrug-resistant (MDR) Gram-negative pathogens from their mothers at or after birth ${ }^{[16]}$ and frequent pathogen exposure through contact with the hospital environment, equipment, staff, parents and other patients. Exposure events may lead to microbial colonisation only or to invasive infection, with consequent morbidity and mortality. Risk factors for neonatal HAI include low birth weight, prematurity, skin fragility, lack of breastfeeding, overcrowding, sharing of equipment and poor adherence to infection-prevention practices. Early exposure to broad-spectrum and prolonged antibiotic courses may permanently alter a neonate's intestinal and skin microbiome and increase the risk of infection. Development of unit-specific antimicrobial treatment protocols based on local data, is considered critical to ensure appropriate empirical antibiotic use and to allow for de-escalation or discontinuation of antibiotics when indicated.

The SA neonatal HAI incidence is likely to rise in the future for several reasons, including predicted population growth with rapid urbanisation, more hospital-based deliveries and immigration from neighbouring countries, exacerbating overcrowding and relative understaffing. Advances in neonatal care have led to ever-smaller babies surviving with prolonged hospital admissions, which confers at least twice the risk of HAI compared with term infants. ${ }^{[17]}$ The rising demand for neonatal services is likely to outstrip the available space, equipment and the ability of public hospitals to maintain ageing infrastructure. Sharing of equipment, shortages of consumables, poor adherence to basic infection-prevention measures and lack of expertise in neonatal unit environmental and equipment cleaning are major challenges. Furthermore, insufficient specialist support and local leadership in infection prevention, hospital epidemiology, infectious diseases, microbiology and antimicrobial and diagnostic stewardships impede development and implementation of local guidelines, limit staff education opportunities and prevent the introduction of sustainable quality-improvement programmes.

\section{The SA Neonatal Sepsis Task Force} and other neonatal infection activities The SA Neonatal Sepsis Task Force was launched at a special plenary session of the United SA Neonatal Association (USANA) conference in Port Elizabeth, SA, on 13 September 2019. Its primary role is to provide technical advice to the national and provincial departments of health and guidance to clinicians on surveillance for neonatal sepsis, prevention, identification and management of cases, and containment of outbreaks. The activities of the task force will focus particularly on prevention and management of AMR infections and/or neonatal HAIs, in public and private sector facilities. The task force is comprised of volunteer members co-opted from professional clinical societies, public hospitals and academia, with expertise in relevant disciplines, including neonatology, paediatric infectious diseases, clinical microbiology, antimicrobial stewardship, infection prevention and control, hospital epidemiology and AMR. The task force will co-ordinate and link activities to other organisations from which members will be drawn, including but not limited 
to: the Federation of Infectious Diseases Societies of Southern Africa (FIDSSA), National Institute for Communicable Diseases (NICD), USANA, Neonatal Nursing Association of SA (NNASA), SA Paediatric Association (SAPA), National Health Laboratory Service (NHLS) and university-affiliated departments across the country. The task force will endeavour to establish and maintain communication channels with relevant departments and committees working with the National Department of Health (NDoH) (e.g. the Ministerial Advisory Committee on AMR, National Perinatal Mortality and Morbidity Committee (NaPeMMCo), Committee on Morbidity and Mortality in Children (CoMMic)) and provincial departments of health.

\section{Infection and outbreak surveillance}

Epidemiological data on all neonatal infections (maternally acquired or early onset v. hospital acquired, sporadic cases v. clusters/outbreaks, bloodstream infections, meningitis and other infection types) are urgently needed from all SA units. This information will assist in developing national guidelines for the management of neonatal infections. It will also assist individual neonatal units and the NDoH to establish the burden of early-onset v. hospitalacquired sepsis and the profile of AMR infections. Furthermore, the data will identify neonatal units with relatively higher infection rates and/or frequent outbreaks for the implementation of qualityimprovement interventions to reduce mortality and healthcare costs. Reported neonatal unit outbreaks in SA are limited, ${ }^{[14,15]}$ although anecdotal reports from clinicians suggest that infection clusters and outbreaks occur frequently. Two externally funded research grants will contribute towards collection of data on neonatal bloodstream infections and outbreaks. Baby GERMS, a Bill and Melinda Gates Foundation-funded project of the NICD, will measure the national burden of neonatal sepsis in SA public sector hospitals and characterise infection risk factors, outcomes and AMR rates at 6 regional neonatal units. The NeoAMR observational cohort is a Global Antibiotic Research and Development Partnership (GARDP)funded, multicountry study of neonatal sepsis patterns and impact, with 3 tertiary hospital sites participating in SA. ${ }^{[18]}$

\section{Infection prevention}

Infection prevention is the cornerstone of high-quality, safe provision of healthcare services to vulnerable populations such as hospitalised and preterm neonates. Despite the acknowledged importance of infection-prevention and control (IPC) programmes, many SA neonatal units lack the staff, infrastructure (water and isolation facilities) and consumables (e.g. alcohol hand-rub, gloves) to support effective IPC. National neonatal quality-improvement strategies include the promotion of breastfeeding, supported by the establishment of milk banks, clean cord care, strict adherence to basic hygiene in labour wards and nurseries, availability of appropriate antibiotic therapy and case management of neonatal sepsis, meningitis and pneumonia. Although these basic infection-prevention measures are important, specialised care and unit-specific infection-prevention protocols are needed where many unique procedures/practices occur, e.g. umbilical catheter placement, surfactant administration, incubator and radiant warmer care, preparation and storage of maternal and donor breast milk and kangaroo mother care. Audits of infection-prevention standards for neonatal units should take into consideration these unique practices, which pose particular infection-prevention challenges. Regular training of all staff, establishing an IPC-neonatal link nurse programme (to support IPC audits and staff training) and appointment of appropriately qualified IPC practitioners are needed for every neonatal unit countrywide. Similarly, efforts to improve IPC implementation and adherence in antenatal and intrapartum care are critical to prevent maternal colonisation and vertical transmission of antibiotic-resistant pathogens. ${ }^{[16]}$ Implementation of the abovementioned processes and interventions should result in reduction in HAI rates and associated morbidity and mortality.

\section{Antimicrobial stewardship}

There is an urgent need for all hospitals to have antimicrobial stewardship programmes (ASPs) as one of the essential steps to conserve the efficacy of currently used antibiotics and delay progression of AMR. To develop strong and effective ASPs, neonatal units must first be assisted to quantify and track trends in their antimicrobial use. Institutional data on pathogen profile and AMR patterns are needed to construct locally appropriate treatment guidelines. Implementation of robust diagnostic and clinical guidelines, improved education of prescribers, feedback of antimicrobial consumption data and AMR trends, as well as strong leadership of the ASP team, are key to reducing inappropriate prescribing and the negative effects of antibiotic exposure in hospitalised neonates. More research is needed to develop and pilot a system for implementing and measuring the impact of ASPs in SA neonatal units.

\section{Conclusions}

The burden of HAIs, AMR and infection outbreaks in SA neonatal units is likely to be higher than the currently available estimates, and may increase further. HAIs and outbreaks are often preventable through provision of high-quality basic neonatal care, appropriate antibiotic use and implementation of IPC best practices, integrated in antimicrobial and diagnostic stewardship programmes. Coordinated national surveillance of neonatal infections and audits of infection-prevention practices and antibiotic use will contribute to identification of high-infection-burden units and prioritise targeted interventions to reduce preventable harm to hospitalised neonates. Greater availability and dissemination of routine data will allow comparison between similar healthcare facilities and establish a baseline for measuring the impact of quality-improvement efforts. Even though the task to be undertaken is huge and includes many challenges, it is critical that this be done as an essential strategy to reduce the high neonatal mortality rate in SA.

\section{Declaration. None.}

Acknowledgements. The authors acknowledge and thank the patients, their parents and the staff of the National Department of Health neonatal units and the staff of the National Health Laboratory Service. Author contributions. AD drafted the first version of the manuscript; all authors contributed to manuscript revisions.

Funding. AD is supported by a US National Institutes of Health (NIH) Fogarty Emerging Global Leader Award K43 TW010682 and receives funding for neonatal infection research from the South African Medical Research Council (self-initiated research grant).

Conflicts of interest. None.

1. Rhoda NR, Velaphi S, Gebhardt GS, Kauchali S, Barron P. Reducing neonatal deaths in South Africa: Progress and challenges. S Afr Med J 2018;108(3a):s9-s16. https://doi.org/10.7196/SAMJ.2018. v108i3.12804

2. Seale AC, Blencowe H, Manu AA, et al., pSBI Investigator Group. Estimates of possible severe bacterial infection in neonates in sub-Saharan Africa, South Asia, and Latin America for 2012: A systematic review and meta-analysis. Lancet Infect Dis 2014;14(8):731-741. https://doi.org/10.1016/S14733099(14)70804-7 
3. Lawn JE, Blencowe H, Oza S, et al., Lancet Every Newborn Study Group. Every newborn: Progress, priorities, and potential beyond survival. Lancet 2014;384(9938):189-205. https://doi.org/10.1016/ proitu-6736(14)60496-7

4. Madhi SA, Pathirana J, Baillie V, et al. Unraveling specific causes of neonatal mortality using minimally Madhi SA, Pathirana J, Baillie V, et al. Unraveling specific causes of neonatal mortality using minimally
invasive tissue sampling: An observational study. Clin Infect Dis 2019;69(S4):S351-S360. https://doi. invasive tissue sampling:

5. World Health Organization. Managing possible serious bacterial infection in young infants when referral is not feasible. Guidelines and WHO/UNICEF recommendations for implementation. Geneva: WHO, 2015 6. Fleischmann-Struzek C, Goldfarb DM, Schlattmann P, et al. The global burden of paediatric and neonatal sepsis: A systematic review. Lancet Respir Med 2018;6(3):223-230. https://doi.org/10.1016//22132600(18)30063-8

7. Chawana R, Baillie V, Izu A, et al. Potential of minimally invasive tissue sampling for attributing specific causes of childhood deaths in South Africa: A pilot, epidemiological study. Clin Infect Dis 2019;69(Suppl 4):S361-S373. https://doi.org/10.1093/cid/ciz550

8. Dramowski A, Madide A, Bekker A. Neonatal nosocomial bloodstream infections at a referral hospital in a middle-income country: Burden, pathogens, antimicrobial resistance and mortality. Paediatr In Child Health 2015;35(3):265-272. https://doi.org/10.1179/2046905515Y.0000000029

9. Crichton H, O'Connell N, Rabie H, Whitelaw AC, Dramowski A. Neonatal and paediatric bloodstream infections: Pathogens, antimicrobial resistance patterns and prescribing practice at Khayelitsha District Hospital, Cape Town, South Africa. S Afr Med J 2018;108(2):99-104. https://doi.org/10.7196/SAMJ.2017. Hospital, Cape
v108i2.12601

10. Ballot DE, Nana T, Sriruttan C, Cooper PA. Bacterial bloodstream infections in neonates in a developing country. ISRN Pediatr 2012;2012:508512. https://doi.org/10.5402/2012/508512
colltallations in

11. Spicer KB, Green J, Dhada B. Hospital-acquired infections in paediatric medical wards at a tertiary hospital in KwaZulu-Natal, South Africa. Paediatr Int Child Health 2018;38(1):53-59. https://doi.org/10 1080/20469047.2017.1299897
12. Ballot DE, Bandini R, Nana T, et al. A review of multidrug-resistant Enterobacteriaceae in a neonatal unit in Johannesburg, South Africa. BMC Pediatr 2019;19(1):320. https://doi.org/10.1186//12887-019-1709-y 13. Velaphi SC, Westercamp M, Moleleki M, et al. Surveillance for incidence and etiology of early-onset nelaphi SC, Westercamp M, Moleeki M, et al. Surveillance for incidence and etiology of early-onset
neonatal sepsis in Soweto, South Africa. PLoS ONE 2019;14(4):e0214077. https://doi.org/10.1371/ neonatal sepsis in So
journal.pone.0214077

14. Dramowski A, Aucamp M, Bekker A, Mehtar S. Infectious disease exposures and outbreaks at a South African neonatal unit with review of neonatal outbreak epidemiology in Africa. Int J Infect Dis 2017;57:79-85. https://doi.org/10.1016/j.ijid.2017.01.026

15. Van Schalkwyk E, Iyaloo S, Naicker SD, et al. Large outbreaks of fungal and bacterial bloodstream infections in a neonatal unit, South Africa, 2012 - 2016. Emerg Infect Dis 2018;24(7):1204-1212. https://doi.org/10.3201/eid2407.171087

16. Bulabula ANH, Dramowski A, Mehtar S. Transmission of multidrug-resistant Gram-negative bacteria from colonized mothers to their infants: A systematic review and meta-analysis. J Hosp Infect 2019;104(1):57-67. https://doi.org/10.1016/.j. hin.2019.10.001

17. Ramasethu J. Prevention and treatment of neonatal nosocomial infections. Matern Health Neonatol Perinatol 2017;3(5). https://doi.org/10.1186/s40748-017-0043-3

18. Li G, Bielicki JA, Ahmed A, et al. Towards understanding global patterns of antimicrobial use and resistance in neonatal sepsis: Insights from the NeoAMR network. Arch Dis Child 2020;105(1):26-31. https://doi.org/10.1136/archdischild-2019-316816

Accepted 17 February 2020. 